\title{
Educación sanitaria al paciente con enfermedad renal crónica avanzada. ¿Existe evidencia de su utilidad?
}

\author{
Francisco Javier Bonilla León \\ Facultad de Medicina y Enfermería. Universidad de Córdoba
}

\section{Resumen}

Introducción: La enfermedad renal crónica se ha convertido en un grave problema sanitario por su elevada incidencia y prevalencia e importante morbimortalidad y coste asistencial. Dada la gravedad del problema, es necesaria una detección precoz de la enfermedad y un tratamiento adecuado de las comorbilidades asociadas. El manejo, por parte del paciente de su tratamiento, dieta, medidas higiénicas, etc, puede prevenir o reducir esta complicación, para lo cual es fundamental la educación sanitaria.

Objetivo: Conocer la producción científica acerca de la utilidad de la educación sanitaria en pacientes con enfermedad renal crónica avanzada.

Métodos: Se trata de una revisión bibliográfica para la que se realizó una búsqueda en las bases de datos de PubMed, Scielo, Science Direct y Google Académico. Se incluyeron artículos científicos escritos en inglés y español. Se analizaron los artículos que trataban la educación sanitaria de pacientes con enfermedad renal crónica avanzada en consultas prediálisis.

Resultados: Tras el análisis de los 24 artículos seleccionados, podemos decir que la educación sanitaria ofrece beneficios tangibles al enfermo renal. Entre las variables desarrolladas, cabe destacar la fuerte implicación que la educación tiene en la elección de modalidad de tratamiento y como se considera un factor asociado a la supervivencia. Además se relaciona con un $17 \%$ menos de riesgo de ingresar en el hospital, un inicio programado de la diálisis, reduce la morbilidad, aumenta los valores de parámetros bioquímicos positivos y da más seguridad al paciente para afrontar el manejo de su enfermedad.

Conclusiones: La educación sanitaria es una herramienta de suma importancia en el tratamiento de los pacien-

Correspondencia:

Francisco Javier Bonilla León

E-mail: fco.bonilla92@gmail.com tes con enfermedad renal crónica avanzada, y se debe ofrecer en el marco de una consulta prediálisis formada por un equipo multidisciplinar, en el que enfermería tiene un rol activo muy importante.

PALABRAS CLAVE:

- EDUCACIÓN SANITARIA

- PREDIÁLISIS

- ENFERMEDAD RENAL CRÓNICA AVANZADA

Health education for patients with advanced chronic kidney disease. Is there evidence of its usefulness?

\begin{abstract}
Introduction: Chronic kidney disease has become a serious health problem for its high incidence and prevalence, significant morbidity and health care costs. Due to the seriousness of the problem, it is required an early detection of the disease and suitable treatment of the associated comorbidities. The management of the patient for his/her treatment, diet, hygienic measures etc. can prevent or reduce this complication, as result health education is fundamental.
\end{abstract}

Purpose: To know scientific production on health education usefulness by advanced chronic kidney disease patients.

Methods: It is a literature review whose search was conducted in the databases of PubMed, Scielo, Science Direct and Google Scholar. Scientific articles written in English and Spanish were included. Articles that dealt with health education programs for advanced chronic kidney disease patients in predialysis clinics were analyzed.

Results: After the analysis of the 24 selected articles, we can state that health education offers tangible benefits to the renal patient. Among the developed variables 
highlighted the strong implication that health education has in choice of treatment modality and it is considered as a factor associated with survival. It is also related to a $17 \%$ reduction in the risk for hospitalizations, a planned dialysis start, reduces morbidity, increases positive biochemical parameters values and increases the confidence of the patient to deal with the management of his disease.

Conclusions: Health education is an important tool in the treatment of patients with advanced chronic kidney disease, and it must be offered in the context of a predialysis clinic that consists of a multidisciplinary team, in which nursing has an important active role.

\section{KEY WORDS:}

- HEALTH EDUCATION

- PREDIALYSIS

- ADVANCED CHRONIC KIDNEY DISEASE

\section{Introducción}

La Enfermedad Renal Crónica (ERC) se define como la disminución de la función renal, expresada por un filtrado glomerular $(F G)<60 \mathrm{ml} / \mathrm{min} / 1,73 \mathrm{~m}^{2}$ o como la presencia de daño renal de forma persistente durante al menos 3 meses. De acuerdo al filtrado glomerular calculado o estimado con distintas fórmulas, se clasifica en los estadios que se recogen en la siguiente tabla.

\begin{tabular}{|c|c|c|}
\hline Estadio & $\left.\mathbf{F G ~ ( m l / m i n / 1 , 7 3} \mathbf{m}^{2}\right)$ & Descripción \\
\hline 1 & $\geq 90$ & Daño renal con FG normal \\
\hline 2 & $60-89$ & Daño renal, ligero descenso del FG \\
\hline 3 & $30-59$ & Descenso moderado del FG \\
\hline 4 & $15-29$ & Descenso grave del FG \\
\hline 5 & $<150$ diálisis & Fallo renal \\
\hline
\end{tabular}

La Enfermedad Renal Crónica Avanzada (ERCA) incluye los estadios 4 y 5, definiéndose como la enfermedad renal crónica que cursa con descenso grave del filtrado glomerular $(\mathrm{FG}<30 \mathrm{ml} / \mathrm{min})^{1-5}$.

La ERCA es un grave problema sanitario, tanto por su elevada incidencia y prevalencia (crecientes en las últimas décadas), como por su importante morbimortalidad y coste asistencial ${ }^{6}$, siendo subsidiaria de tratamiento renal sustitutivo (TRS).

Según los resultados preliminares del estudio EPIRCE (Epidemiología de la Insuficiencia Renal Crónica en
España), aproximadamente el $11 \%$ de la población adulta sufre algún grado de ERC, con una tasa elevada de factores de riesgo vascular asociados y un progresivo incremento del número de pacientes subsidiarios de depuración extrarrenal, estimado en un 5-8\% anual ${ }^{7}$. Esto se debe a que sus causas principales residen en trastornos de alta prevalencia como el envejecimiento, la hipertensión arterial, la diabetes y la enfermedad vascular ${ }^{8}$. El coste anual asociado al tratamiento de las fases más avanzadas de ERC se estima en más de 600 millones de euros?.

Con esta situación y las previsiones futuras probablemente la única estrategia realista para abordar el problema es realizar un esfuerzo de detección precoz de la ERC y de los pacientes de riesgo para desarrollar enfermedad renal, así como una prevención y tratamiento adecuado de las comorbilidades asociadas ${ }^{10}$.

De forma programada, los pacientes llegan a la consulta ERCA, Ilamada también de Prediálisis o bajo aclaramiento en el estadio 3.

Esta consulta, además del seguimiento y control de la función renal, evolucionó desde hace unos años hacia el concepto de cuidado integral del paciente renal en esta etapa. Dentro del cual, se incorporó la educación sanitaria, realizada por enfermería y con programas cada vez más protocolizados ${ }^{11}$. Este proceso de educación sanitaria permite llevar un seguimiento más constante de los pacientes, a los que se les da información de diversa temática relacionada con la patología, como dieta, accesos vasculares, posibles problemas inter y post-hemodiálisis, turnos y horarios, transporte... Esta información se les da de forma reglada y paulatina, y se va ampliando a medida que el paciente va asumiendo su proceso ${ }^{12}$.

La Consulta ERCA o Prediálisis favorece la atención individualizada de los pacientes con ERCA, al ayudar al paciente y su familia a tomar una decisión informada respecto al procedimiento de diálisis; la estandarización y protocolización del seguimiento; la implantación con suficiente antelación de un acceso o catéter idóneo; la vacunación contra la Hepatitis B y en definitiva, la mejora en la eficiencia (costes), la disminución de la morbimortalidad y el incremento en el bienestar de los pacientes ${ }^{13}$.

El objetivo principal de la consulta de Prediálisis, por tanto, es proporcionar un cuidado integral del paciente con ERCA, en el que se contemple la asistencia, información, formación, educación e investigación consensuada. 
Los objetivos parciales prioritarios son:

\begin{abstract}
- Asistencial, con actuaciones centradas en promover la referencia precoz al nefrólogo, retrasar la progresión de la ERC, prevenir y tratar las complicaciones de la ERCA, modificar o tratar la comorbilidad asociada y coordinarse con la Unidad de Cuidados Paliativos, en el caso de que el paciente requiera solo tratamiento conservador ${ }^{14}$.

- Información al paciente e inclusión del mismo en un Programa de Formación o Educación progresiva en autocuidados: Información sobre la ERC y sus posibilidades de tratamiento para una elección independiente, y preparación para inicio de TRS.
\end{abstract}

- Análisis de Calidad Asistencial, mediante el seguimiento de marcadores de calidad asistencial directos, calidad de vida y satisfacción del usuario ${ }^{15}$.

Por todo ello, el papel de enfermería, como parte del equipo multidisciplinar que debería formar la consulta prediálisis, es fundamental, como responsable de impartir una adecuada educación sanitaria a estos pacientes.

El comité de Expertos de la OMS define la Educación para la Salud (EPS) como un proceso interdisciplinar, dinámico y multiprofesional, influenciado por factores externos que conforman el entorno del individuo dentro de la sociedad, tendentes a lograr un mayor nivel de salud mediante la adopción de hábitos y estilos de vida saludables. A pesar de esto, la EPS es, aun hoy, una de las parcelas en las que menos recursos se invierten a todos los niveles del sistema ${ }^{16,17}$.

En la actualidad la única patología en la que se realiza un programa de educación sanitaria completo y con buenos resultados es la diabetes. En los estudios que evalúan los programas de educación sanitaria en pacientes diabéticos se evidenciaron numerosas mejoras en el grupo que recibió la educación con respecto al grupo control, como por ejemplo, aumento de conocimientos, cambios en el estilo de vida, más control glucémico, reducción de los ingresos hospitalarios con mejora del coste de la atención, retraso en la aparición de complicaciones y cambios positivos de los indicadores clínicos ${ }^{18-22}$.

En relación a la diabetes, está demostrado que la EPS tendrá más efecto en los pacientes recién diagnosticados y que aun no han desarrollado complicaciones crónicas ${ }^{23}$, y que realizándola en grupo surge más efecto que de forma individual ${ }^{24,25}$. Esta EPS fue asumida por enfermería estando institucionalizada en todos los centros sanitarios de nuestro entorno, formando actualmente parte indispensable del cuidado integral del paciente diabético ${ }^{26}$.
Sin embargo, no existen muchas evidencias que justifiquen la necesidad de implantación de programas de EPS en otras áreas o en pacientes con otras alteraciones crónicas. En efecto, se utiliza mucho en el argot profesional de enfermería la necesidad de educación sanitaria en casi todas las áreas asistenciales, como necesidad de justificar este rol profesional, aunque la realidad es que esta EPS se realiza sin la estructura pedagógica adecuada y sobre todo, sin evaluar su eficacia ${ }^{27}$.

Es por ello, por lo que nos hemos planteado este estudio, pues aunque las estrategias educativas han sido utilizadas para la mejora y aumento de los conocimientos en distintas patologías, favoreciendo la adhesión al tratamiento por parte del paciente y familia, y la calidad de vida que mantienen, no existen muchos estudios que avalen este hecho en pacientes con ERC.

Una buena educación sanitaria es beneficiosa e involucra a los pacientes con patología renal en sus autocuidados, logrando que el paciente consiga una mejor aceptación de su enfermedad, que se adapte a la nueva situación biopsicosocial, que cumpla mejor el tratamiento, que tenga mayor criterio en la elección de la técnica y por tanto conseguir un mejor pronóstico en la evolución de su enfermedad ${ }^{28}$.

Debido a la importancia de la ERCA y su repercusión en el estilo y calidad de vida de los enfermos, es imprescindible conocer si la existencia de una consulta de prediálisis ofrece beneficios a los pacientes.

Por tanto, el objetivo de la presente revisión fue conocer la producción científica acerca de la utilidad de la educación sanitaria en pacientes con ERCA.

Objetivo específico: Identificar las variables relacionadas con la educación sanitaria en el paciente con ERCA y su influencia en resultados en salud.

\section{Metodología}

El diseño empleado fue el de un estudio de revisión bibliográfica, para la que se realizó una búsqueda en diversas bases de datos con el objetivo de recopilar la mayor información posible sobre el tema tratado.

Las bases de datos consultadas fueron: Pubmed, Scielo, Science Direct y Google Académico. El periodo de consulta de las bases de datos tuvo lugar desde el 25 de Marzo de 2014 hasta el 10 de Mayo de 2014.

La búsqueda incluyó artículos en español e inglés, y el método de búsqueda consistió en utilizar como palabras 
clave en las bases de datos: educación sanitaria (health education); prediálisis (predialysis); y enfermedad renal crónica avanzada (advanced chronic kidney disease) unidos dichos términos por los operadores boleanos "and" $y$ "or", y empleando los distintos descriptores en singular, plural y que los descriptores estuvieran presentes en el resumen, título del artículo y, si la base de datos lo presenta, en palabras clave. Se acotó la búsqueda a aquellos artículos que tenían disponible el texto completo.

\section{Los criterios de inclusión fueron:}

- Artículos en los cuales se trataran programas de educación sanitaria en pacientes en fase de prediálisis o consulta ERCA.

- Trabajos relacionados con la actividad de enfermería en esta etapa.

- Artículos originales completos.

\section{Los criterios de exclusión fueron:}

- Trabajos que no presentaban resultados.

- Artículos que trataran sobre programas educativos en otras fases de la enfermedad renal crónica.

Al inicio de la búsqueda no se tuvo en cuenta los criterios de inclusión y exclusión, para ver la cantidad de producción científica existente en el tema y obtener mayor conocimiento.

Después de recopilar los artículos, se procedió a la lectura de todos los títulos y resúmenes para descartar aquellos que no seguían los criterios de inclusión/ exclusión, no tuvieran relación con el tema tratado o estuvieran repetidos.
Posteriormente se procedió a la lectura del texto completo de los artículos seleccionados para incluirlos en el trabajo.

\section{Resultados}

En función de las distintas bases de datos, los resultados obtenidos y el método de búsqueda variaron. En la tabla I, se puede observar los documentos localizados en cada base de datos.

Tabla I. Número de publicaciones encontradas y preseleccionadas en cada base

\begin{tabular}{|l|c|c|c|c|c|}
\hline Base de datos & Pubmed & Scielo & $\begin{array}{c}\text { Science } \\
\text { Direct }\end{array}$ & $\begin{array}{c}\text { Google } \\
\text { Académico }\end{array}$ & Total \\
\hline $\begin{array}{c}\text { Publicaciones } \\
\text { encontradas }\end{array}$ & 103 & 30 & 12 & 32 & 177 \\
\hline $\begin{array}{c}\text { Publicaciones } \\
\text { preseleccionadas }\end{array}$ & 33 & 24 & 9 & 17 & 83 \\
\hline
\end{tabular}

En total, de los 83 documentos preseleccionados de todas las bases de datos, tras eliminar los repetidos (8) y los que no se ajustan al estudio (11), se quedaran 64 artículos que cumplían con los criterios de inclusión/exclusión establecidos en este estudio de revisión.

En la segunda revisión tras hacer una lectura completa de todos los artículos volvimos a excluir alguno más, porque no encajaban del todo con el objetivo de nuestra revisión, dejando así finalmente los que aparecen en la bibliografía, que quedan reflejados en la tabla II.

Tabla II. Clasificación de todos los artículos seleccionados para el trabajo

\begin{tabular}{|c|c|c|c|}
\hline Autor, año, país & Muestra & Diseño del & Variables estudiadas \\
\hline $\begin{array}{l}\text { Marrón B, Martínez JC, } \\
\text { Salguiera M et al. } \\
\text { 2005. Países Bajos }{ }^{38}\end{array}$ & 621 & Multicéntrico, retrospectivo & $\begin{array}{l}\text { Elección de modalidad de tratamiento, } \\
\text { seguimiento médico, inicio de la diálisis, } \\
\text { parámetros bioquímicos }\end{array}$ \\
\hline $\begin{array}{l}\text { Choi ES, Lee J. } \\
\text { 2012. Korea }{ }^{29}\end{array}$ & 61 & Control, no equivalente, no sincronizado & $\begin{array}{l}\text { Nivel de conocimientos, práctica de } \\
\text { autocuidado, parámetros bioquímicos }\end{array}$ \\
\hline $\begin{array}{c}\text { Lacson E, Wang W, De Vries C et al. } \\
\text { 2011. EEUU }{ }^{38}\end{array}$ & 20.057 & Observacional, multicéntrico & $\begin{array}{l}\text { Mortalidad, supervivencia, elección de } \\
\text { modalidad de tratamiento }\end{array}$ \\
\hline $\begin{array}{l}\text { Rognant N, Alamartine } \mathrm{E}_{\text {, }} \\
\text { Aldigier JC et al. } \\
\text { 2013. Francia }{ }^{51}\end{array}$ & 160 & $\begin{array}{c}\text { Observacional, cohorte, multicéntrico, } \\
\text { prospectivo }\end{array}$ & $\begin{array}{c}\text { Mortalidad, ingresos hospitalarios, se- } \\
\text { guimiento médico, medicación prescrita, } \\
\text { parámetros bioquímicos }\end{array}$ \\
\hline
\end{tabular}




\section{[ Francisco Javier Bonilla León ]}

Educación sanitaria al paciente con enfermedad renal crónica avanzada. ¿Existe evidencia de su utilidad?

\begin{tabular}{|c|c|c|c|}
\hline Autor, año, país & Muestra & Diseño del & Variables estudiadas \\
\hline $\begin{array}{l}\text { Gago MC, Martínez S, } \\
\text { Sesmero C et al. } \\
\text { 2007. España }{ }^{39}\end{array}$ & 198 & Prospectivo & $\begin{array}{l}\text { Elección modalidad de tratamiento, pará- } \\
\text { metros bioquímicos, morbilidad, mortalidad, } \\
\text { supervivencia, vacunación, inicio de la diálisis }\end{array}$ \\
\hline $\begin{array}{l}\text { Jungers } P \text {, Massy ZA, } \\
\text { Nguyen-Khoa T. } \\
\text { 2001. Francia }{ }^{48}\end{array}$ & 1.057 & Observacional, retrospectivo & $\begin{array}{l}\text { Supervivencia, morbilidad, mortalidad, } \\
\text { parámetros bioquímicos }\end{array}$ \\
\hline $\begin{array}{l}\text { Goldstein M, Yassa T, } \\
\text { Dacouris N et al. } \\
\text { 2004. Canadá }{ }^{44}\end{array}$ & 87 & Cohorte, retrospectivo & $\begin{array}{l}\text { Elección modalidad de tratamiento, inicio de la } \\
\text { diálisis, estado clínico, parámetros bioquímicos, } \\
\text { mortalidad, ingresos hospitalarios, supervivencia }\end{array}$ \\
\hline $\begin{array}{l}\text { Wu IW, Wang SY, Hsu KH et al. } \\
\text { 2009. Taiwán }{ }^{41}\end{array}$ & 573 & Cohorte, controlado, no aleatorizado & $\begin{array}{l}\text { Mortalidad, supervivencia, ingresos } \\
\text { hospitalarios, elección de modalidad de } \\
\text { tratamiento }\end{array}$ \\
\hline $\begin{array}{l}\text { Devins GM, Barré PE et al. } \\
\text { 2005. Canadá }{ }^{30}\end{array}$ & 335 & Multicéntrico, controlado, aleatorizado & $\begin{array}{l}\text { Nivel de conocimientos, supervivencia, } \\
\text { estado clínico }\end{array}$ \\
\hline $\begin{array}{l}\text { Portela L, Menéndez A, } \\
\text { Bermúdez C et al. } \\
\text { 2001. España }\end{array}$ & 286 & Observacional, descriptivo & $\begin{array}{c}\text { Elección de modalidad de tratamiento, } \\
\text { nivel de conocimientos }\end{array}$ \\
\hline $\begin{array}{c}\text { White CA, Pilkey RM, Lam M, } \\
\text { Holland DC. } \\
\text { 2002. Reino Unido }\end{array}$ & 120 & Cohorte, retrospectivo & $\begin{array}{l}\text { Parámetros bioquímicos, ingresos } \\
\text { hospitalarios, mortalidad, estado clínico }\end{array}$ \\
\hline $\begin{array}{l}\text { Manns BJ, Taub K, Jones H et al. } \\
\text { 2005. Canadá32 }\end{array}$ & 70 & Cohorte, aleatorizado, controlado & $\begin{array}{l}\text { Nivel de conocimientos, elección de } \\
\text { modalidad de tratamiento }\end{array}$ \\
\hline $\begin{array}{c}\text { Chen SH, Tsai YF, Sun CY et al. } \\
\text { 2011. Taiwán }\end{array}$ & 54 & Control, aleatorizado & $\begin{array}{l}\text { Ingresos hospitalarios, parámetros } \\
\text { bioquímicos, mortalidad }\end{array}$ \\
\hline $\begin{array}{l}\text { Curtis BM, Ravani } P \text {, } \\
\text { Malberti F et al. } \\
\text { 2005. Canadá42 }\end{array}$ & 288 & Control & $\begin{array}{l}\text { Elección de modalidad de tratamiento, } \\
\text { parámetros bioquímicos, supervivencia }\end{array}$ \\
\hline $\begin{array}{l}\text { Pulido F, Arribas E, } \\
\text { González F et al. } \\
\text { 2009. España }{ }^{35}\end{array}$ & 411 & Prospectivo & $\begin{array}{l}\text { Parámetros bioquímicos, elección de mo- } \\
\text { dalidad de tratamiento, vacunación, nivel } \\
\text { de conocimientos, ingresos hospitalarios }\end{array}$ \\
\hline $\begin{array}{l}\text { Yang LM, Han TC, Lin PL et al. } \\
\text { 2013. Taiwán }{ }^{33}\end{array}$ & 248 & Cuasi-experimental & $\begin{array}{l}\text { Nivel de conocimientos, práctica de } \\
\text { autocuidado, seguimiento médico }\end{array}$ \\
\hline $\begin{array}{c}\text { Marrón B, Ortiz A, } \\
\text { de Sequera } P \text { et al. 2006. España }{ }^{34}\end{array}$ & 1.504 & Multicéntrico, retrospectivo & $\begin{array}{l}\text { Inicio de la diálisis, parámetros bioquí- } \\
\text { micos, nivel de conocimientos, elección } \\
\text { de la modalidad de tratamiento }\end{array}$ \\
\hline $\begin{array}{c}\text { Gutiérrez JM, Ibars P, Pitarch G. } \\
\text { 2002. España }{ }^{36}\end{array}$ & 19 & Observacional, analítico & $\begin{array}{l}\text { Elección de la modalidad de } \\
\text { tratamiento, nivel de conocimientos }\end{array}$ \\
\hline $\begin{array}{l}\text { Devins GM, Mendelssohn DC, } \\
\text { Barré PE et al. } \\
\text { 2003. Canadá37 }\end{array}$ & 297 & Cohorte, prospectivo, controlado, aleatorizado & $\begin{array}{l}\text { Nivel de conocimientos, parámetros } \\
\text { bioquímicos, inicio de la diálisis }\end{array}$ \\
\hline $\begin{array}{l}\text { Goovaerts T, Jadoul M, Goffin E. } \\
\text { 2005. Bélgica }{ }^{45}\end{array}$ & 235 & Retrospectivo & $\begin{array}{l}\text { Elección de modalidad de tratamiento, } \\
\text { inicio de la diálisis }\end{array}$ \\
\hline $\begin{array}{c}\text { Harris LE, Luft FC, Rudy DW et al. } \\
\text { 1998. EEUU }{ }^{49}\end{array}$ & 437 & Control, aleatorizado & $\begin{array}{l}\text { Seguimiento médico, ingresos hospitala- } \\
\text { rios, medicación prescrita, mortalidad }\end{array}$ \\
\hline $\begin{array}{c}\text { Levin A, Lewis M, Mortiboy P et al. } \\
\text { 1997. Canadá46 }\end{array}$ & 76 & Cohorte, prospectivo, aleatorizado & $\begin{array}{c}\text { Inicio de diálisis, elección de modalidad } \\
\text { de tratamiento, ingresos hospitalarios, } \\
\text { parámetros bioquímicos }\end{array}$ \\
\hline $\begin{array}{c}\text { Gómez AC, Fernández D, et al. } \\
\text { 2011. España }{ }^{43}\end{array}$ & 151 & Observacional, transversal, retrospectivo & $\begin{array}{l}\text { Elección de modalidad de tratamiento, } \\
\text { mortalidad }\end{array}$ \\
\hline
\end{tabular}




\section{Descripción de los resultados:}

Tras leer los artículos completos y analizar los resultados de cada uno de ellos, destacamos las siguientes variables:

\section{Nivel de conocimientos}

Según varios estudios $29-34$, los pacientes que participan en un programa de educación sanitaria específico (consulta multidisciplinaria prediálisis) tienen un aumento en el nivel de conocimientos con respecto a los pacientes que no reciben dicha educación (consulta nefrológica estándar).

Además, se ha demostrado que el aumento de conocimientos sobre los distintos aspectos de la enfermedad (causas, tratamiento, dieta...) disminuye el temor y la ansiedad, y aumenta las expectativas del tratamiento ${ }^{35,36}$.

Otro estudio ${ }^{37}$, encuentra una relación entre el aumento de conocimientos y una mayor duración de la terapia de diálisis.

\section{Elección de modalidad de tratamiento}

Uno de los aspectos más estudiados y analizados es la elección de la modalidad de tratamiento. Todas las intervenciones educativas dedican gran parte de su tiempo a informar sobre las diferentes modalidades de tratamiento: tratamiento conservador y tratamiento sustitutivo, que engloba hemodiálisis (HD), diálisis peritoneal (DP) y trasplante renal (TR), siendo el tratamiento más elegido la $\mathrm{HD}^{35,36,38-43}$.

La educación sobre los tipos de modalidades existentes se asocia con un aumento en la elección de DP $31,34,35,38,39,41,43-46$, pero la HD sigue siendo el tratamiento más elegido35,36,38,41,43,44. Por tanto, la consulta ERCA se convierte en un factor asociado a la elección de $D P$, debido a que los pacientes que no han recibido información sobre esta modalidad no la eligen por miedo y desconocimiento.

Sólo hay un estudio ${ }^{34}$, en el que los pacientes que han recibido educación sanitaria escogen más DP que HD.

El tratamiento conservador no fue elegido por muchos pacientes, aunque en uno de los estudios ${ }^{43}$ fue escogido por el $23,2 \%$ de la muestra.

Los pacientes provenientes de la consulta prediálisis eligen más el tratamiento de diálisis en domicilio, debido a que se les informa sobre los mejores resultados, menos riesgos, mayor satisfacción y menor coste $32,40,45$.

La elección de modalidad se relaciona con la causa de la ERCA y la edad, siendo la elección de HD en domicilio inversamente proporcional a la edad de los pacientes ${ }^{45}$.
En cuanto al TR, en el artículo de Curtis BM, Ravani $P$, Malberti $\mathrm{F}$ et al. ${ }^{42}$ se identifican más pacientes que eligen este tratamiento entre el grupo que recibe consulta nefrológica estándar, no consulta multidisciplinaria.

\section{Inicio de la diálisis}

La educación sanitaria se ha relacionado con un inicio programado de la diálisis. En la mayoría de estudios, un alto porcentaje de los pacientes que provienen de consultas ERCA, inician la diálisis de forma programada, mientras que de los pacientes que lo hacen de forma urgente, muy pocos han recibido educación sanitaria $34,38,39,45,46$.

Los pacientes que inician el tratamiento de diálisis de forma programada, lo suelen hacer con acceso vascular o peritoneal permanente $34,38,44$.

Se ha demostrado que iniciar de forma urgente y sin acceso permanente la diálisis, aumenta la morbimortalidad, la mortalidad y los costes de la asistencia, por lo que la educación sanitaria es fundamental en este sentido ${ }^{38}$.

Además, otros artículos demuestran que una intervención psicoeducativa en la época de prediálisis retrasa el inicio de la diálisis ${ }^{37,45}$.

\section{Mortalidad y supervivencia}

La relación entre programas educativos y mortalidad aparece en la mayoría de artículos, siendo más alta la tasa de mortalidad entre los pacientes que no participan en dichos programas $39-41,43,44,48-50$.

Sin embargo, otros estudios ${ }^{51,52}$ tuvieron porcentajes muy similares de fallecimiento entre ambos grupos.

En todos los estudios se identificaron las mismas causas de muerte en ambos grupos de pacientes.

En un estudio ${ }^{47}$ se asoció la consulta con un $44 \%$ menos de riesgo de muerte, y otro ${ }^{44}$ identificó como principales factores de riesgo no provenir de la consulta ERCA, tener edad avanzada al inicio de la diálisis y presencia de trastorno cardiovascular.

Varios estudios $30,39,40,42,47,48$ demuestran que la supervivencia es mayor en los pacientes que han participado en algún programa educativo. En el estudio de Goldstein M, Yassa T, Dacouris N, McFarlane P. ${ }^{44}$ la supervivencia es mayor en los pacientes de la consulta durante los primeros seis meses de tratamiento, mientras que tras este tiempo se iguala. Por el contrario, otro estudio ${ }^{41}$ identificó la misma supervivencia para ambos grupos de pacientes.

Se ha identificado la procedencia de la consulta prediálisis como un factor asociado a la supervivencia ${ }^{39}$. 


\section{Morbilidad}

La principal causa de morbimortalidad en los pacientes con ERC en cualquiera de sus fases es la enfermedad cardiovascular. A pesar de ser un importante problema, sólo se analiza en dos estudios. En el primero, se demuestra que una temprana referencia a la consulta de prediálisis y una estancia prolongada en ella, reduce el riesgo de aparición de problemas cardiovasculares ${ }^{48}$.

Sin embargo, el índice de comorbilidad de Charlson es igual en todos los pacientes estudiados por el otro artículo ${ }^{39}$, pertenezcan o no a la consulta.

\section{Ingresos hospitalarios}

Los pacientes que participan en programas educativos tienen una tasa más baja de hospitalizaciones, tanto antes como durante el tratamiento de diálisis 35,41,44,46,50-52.

En otro artículo ${ }^{47}$ se relaciona la asistencia a consultas multidisciplinarias con un $17 \%$ menos de riesgo de ingresar en el hospital, aunque no se considera una diferencia significativa. En cuanto a las causas de los ingresos, se han observado las mismas para todos los pacientes ${ }^{47,52}$.

No hay evidencia clara sobre el número de días que los pacientes permanecen ingresados, debido a que no se ha estudiado en la mayoría de artículos analizados. En uno se produjeron los mismos días para todos los pacientes, hubieran o no recibido educación ${ }^{49}$. Por el contrario, en otro se produjo el doble de días en los pacientes que no recibieron educación en la etapa prediálisis ${ }^{46}$.

\section{Seguimiento médico}

Diversos estudios relacionan los programas de educación sanitaria con un mayor seguimiento, tanto médico como específico $38,49,51$.

El hecho de realizar más visitas médicas y tener más control por parte de los profesionales sanitarios hace que la progresión de la enfermedad renal sea más lenta y la calidad de vida del paciente mayor ${ }^{33,51}$.

\section{Parámetros bioquímicos}

En varios artículos $29,34,38,46,51,52$, la tasa de filtrado glomerular es parecida en todos los pacientes al principio, pero cuando se va a iniciar la diálisis, la tasa es más alta en los pacientes que provienen de la consulta. Además, en el mismo periodo de tiempo la reducción del filtrado glomerular es mucho más alta en el grupo no intervenido, lo que indica que la consulta prediálisis influye en la ralentización de la progresión de la enfermedad $^{52}$. Otros artículos 37,42 , sin embargo encontraron que la función renal era igual en ambos grupos.
En cuanto a los niveles de hemoglobina, tres artícu$\operatorname{los}^{29,39,42}$ reflejan mejores niveles en el grupo que recibió la intervención, aunque en uno de ellos ${ }^{29}$ no hubo diferencias significativas.

Los niveles de sodio $^{29}$, albumina sérica y de calcio sí fueron considerablemente más altos en el grupo prediálisis ${ }^{39,42,44}$.

La función renal residual (medida por el aclaramiento de creatinina residual) fue más alta en el grupo prediálisis al inicio de la terapia renal sustitutiva, según dos estu$\operatorname{dios}^{35,50}$. Al igual, en otros artículos ${ }^{46,52}$ se identificó un nivel de creatinina sérica más alto en los pacientes control.

Otro factor que se asocia a unos mejores valores bioquímicos es el inicio programado de la diálisis ${ }^{38,48}$.

\section{Práctica de autocuidados}

En el estudio de Choi Es y Lee $J^{29}$ la práctica de autocuidados fue aumentando según avanzaba el programa educativo, mientras que en el grupo control no varió. Según este estudio, para mejorar esta variable es más eficaz la educación individualizada en pequeños grupos, porque hay más interacción paciente-profesional sanitario.

En otro estudio ${ }^{33}$, también queda reflejado que los programas educativos mejoran los autocuidados, la actitud del paciente y sus estilos de vida.

\section{Medicación prescrita}

Un estudio ${ }^{49}$ no encontró suficiente diferencia significativa en sus datos, los cuales sólo diferenciaban que el grupo atendido por un equipo multidisciplinar tuvo menos uso de clonidina (antihipertensivo) y suplementos de potasio, pero más prescripción de bloqueadores de canales de calcio y beta-bloqueadores en general.

Otro estudio ${ }^{51}$, encontró algunas diferencias en el uso de varios fármacos. Describió un uso parecido en fármacos antihipertensivos, antiplaquetarios, hierro (vía oral e intravenosa), suplementos de potasio y protectores cardiacos y renales. Sin embargo, encontró que el grupo control (sin recibir educación sanitaria) tomaba más inhibidores del sistema renina-angiotensina, betabloqueadores, eritropoyetina y tratamiento óseo.

En cuanto a la adherencia al tratamiento, según algunos estudios ${ }^{41,52}$ en el grupo intervenido hubo un aumento de la misma.

\section{Vacunación}

Los pacientes con insuficiencia renal crónica están en mayor riesgo de contraer infecciones por virus de la hepatitis $B$, debido a la alteración de la inmunidad re- 
lacionada con la uremia, las prácticas terapéuticas invasivas y el tratamiento inmunosupresor tras el trasplante. Los pacientes con ERC tienen indicadas tres vacunas: la de la hepatitis B, la de la gripe y la anti-neumocócica.

En los artículos analizados, sólo se hace referencia a la vacunación de la hepatitis $B$ en dos de ellos ${ }^{35,39}$, indicando que un alto porcentaje de los pacientes que pertenecen a la consulta prediálisis tienen puesta la vacuna. No obstante, no existe comparación con el grupo control por lo que no está demostrada la relación entre educación sanitaria y vacunación.

Es importante la vacunación en estos pacientes en fases tempranas de la enfermedad, ya que la capacidad de producir anticuerpos se correlaciona con el grado de función renal.

\section{Estado clínico}

Varios son los artículos que confieren una mayor salud a los pacientes pertenecientes a la consulta prediálisis que a los que reciben una atención nefrológica estándar ${ }^{30,44,50}$. Además, los pacientes que son captados de forma tardía a la consulta tienen una peor salud que los captados de forma temprana ${ }^{30}$.

Al inicio del tratamiento renal sustitutivo, todos los pacientes (grupo control y experimental) tenían las mismas constantes vitales en uno de los estudios ${ }^{44}$. Sin embargo no hay referencia en ningún otro artículo.

En el estudio de White CA, Pilkey RM, Lam M y HoIland $\mathrm{DC}^{50}$, la consulta prediálisis fue asociada con una mejor función física, función social y menos limitación del rol emocional del paciente. Además, se asoció con unas mejores puntuaciones en diversas cuestiones referidas a la salud en general.

La asistencia a la consulta prediálisis es un predictor independiente de la mejora de calidad de vida en los primeros seis meses después del inicio de la diálisis ${ }^{30,50}$.

\section{Discusión}

En esta revisión se ha puesto de manifiesto que la inclusión de los pacientes con ERCA en un programa de educación sanitaria ofrece beneficios en múltiples áreas.

En los estudios revisados existen diferentes enfoques en cuanto a la forma de impartir la educación sanitaria, dependiendo de varios factores. Aunque queda manifestado que es más útil realizar sesiones interactivas con pocos pacientes para que exista una buena relación terapéutica, y utilizar folletos, videos y demás material didáctico para mejorar el conocimiento ${ }^{30,32}$.
A la hora de medir los resultados, se utilizaron diferentes herramientas dependiendo de qué evaluaba cada estudio. Algunos artículos, evaluaban el resultado de intervenciones educativas mediante parámetros y mediciones clínicas objetivas $38,39,46,51$. Mientras que otros estudios de investigación psicológica, se centraban en evaluar conocimientos y factores de comportamiento a través de cuestionarios $^{29-31}$.

Uno de los hallazgos de este trabajo, expresado por todos los artículos, es como a través de la educación sanitaria, el nivel de conocimientos de los pacientes aumentó notablemente ${ }^{29-34}$. Esto influye en el resto de variables estudiadas con más o menos efecto.

La mayoría de estudios relacionan la educación y la elección de la modalidad de tratamiento, aunque se centran en la modalidad de diálisis. No hay duda de que el tratamiento de primera elección es la hemodiálisis ${ }^{35,36,38-43}$, tanto en los pacientes "educados" como en los que no lo han sido. El único estudio con resultados que no concluyen en esto es el de Marrón B et al34, cuyos pacientes "educados" optaron en mayor proporción por la diálisis peritoneal. No obstante, varios artículos han descrito a la consulta prediálisis como un factor asociado a la elección de diálisis peritoneal ${ }^{31,34,35,38,39,41,43-46}$. Aunque la educación ofrecida por la consulta Prediálisis o ERCA es un importante factor que afecta a la elección de una modalidad u otra, también influyen otros, como la edad de los pacientes ${ }^{45}$.

Con respecto al inicio de la diálisis, según Marrón et al ${ }^{38}$. en su artículo, que analiza el flujo de pacientes a diálisis y cómo influye la educación en la elección de modalidad, se evidencia que iniciar de forma urgente el proceso de diálisis trae consecuencias negativas, tanto para el paciente (aumento de la mortalidad, morbilidad...), como para el sistema (aumento de los costes). Al relacionarse el inicio programado con la educación sanitaria, los resultados son positivos en todos los estudios 34,38,39,45,46, por lo que, parece razonable que la implantación de programas de educación sanitaria podría prevenir parte de los problemas anteriormente citados.

Otro hallazgo del estudio es la relación directa entre educación sanitaria y disminución de la mortalidad y morbilidad. En este campo, hay discrepancias entre los artículos. Mientras la mayoría indican que la tasa de mortalidad es más alta en pacientes que no han sido "educados" 39-41,43,44,47-50, los estudios de Rognant et al ${ }^{51}$. y Chen et $\mathrm{al}^{52}$. encontraron porcentajes similares de fallecimientos entre los dos grupos de pacientes. Se debe tener en cuenta las limitaciones que presentaban dichos 
artículos, los cuales cuentan con un tamaño muestral pequeño, un corto seguimiento ${ }^{52}$, y un tipo de diseño erróne $0^{51}$. Sobre la morbilidad, está reconocido que la principal causa de morbimortalidad en los pacientes con enfermedad renal es la enfermedad cardiovascular $39,48,53$

Con respecto a las hospitalizaciones, los pacientes que asistieron a programas educativos tuvieron un número de ingresos inferior al grupo que no lo hizo $3,41,44,46,50-52$, por lo que Hemmelgarn et $\mathrm{al}^{47}$, relacionó la asistencia a consultas multidisciplinarias con un $17 \%$ menos de riesgo de ingresar en el hospital. En cuanto a las causas de dichos ingresos hospitalarios fueron las mismas para todos los pacientes y principalmente se debieron a problemas cardiovasculares, complicaciones de la diabetes e infecciones.

La asistencia a consultas médicas específicas (oftalmología, dietética...) fue mayor en los pacientes "educados $^{\prime \prime}$, debido a que se generó en ellos un mayor interés por su enfermedad y por tener la mayor calidad de vida posible, evitando la aparición de complicaciones. Este factor también lo vemos en la práctica de autocuidados, que aumentó notablemente en pacientes provenientes de la consulta ${ }^{29,33}$.

Otro hallazgo fue como los parámetros bioquímicos fueron mejores tras los programas educativos, mejorando el aclaramiento de creatinina, los niveles de sodio, hemoglobina y albúmina, y la tasa de filtrado glomeru$\operatorname{lar}^{29,34,38,44,46,51,52}$. No obstante, hubo dos artículos ${ }^{37,42}$ que indicaron una función renal igual para todos los pacientes estudiados.

Finalmente, también se han identificado otras variables en las que la educación tiene un importante papel: la vacunación y el estado clínico del paciente.

Referente a la vacunación, sólo es descrita en dos artículos $^{35,39}$ en los que se ve un alto porcentaje de pacientes de la consulta vacunados frente al virus de la hepatitis $B$, pero no son datos significativos para nuestro trabajo puesto que no están comparados con el porcentaje de vacunados en el grupo control, por lo que no podemos asegurar que la educación para la salud haya influido en esta variable.

El estado clínico mejoró en los pacientes que habían recibido educación sanitaria, demostrando una mejor función física, social y menos limitación del rol emocional, según el estudio de White et $\mathrm{al}^{50}$.

Con estos resultados, se puede afirmar que una temprana referencia a la consulta prediálisis donde el personal de enfermería ofrezca educación sanitaria en el marco de un equipo multidisciplinar podría reducir la incidencia de pacientes que inician de forma urgente su tratamiento en diálisis, reducir el temor, mejorar la calidad de vida de los pacientes, entre otras ventajas.

Varios de los artículos no solo evalúan la utilidad de la consulta prediálisis, sino que también valoran si la labor de un equipo multidisciplinario ofrece más beneficios.

Los equipos multidisciplinarios son una estrategia sugerida hace ya más de un decenio por grupos canadienses. Los pacientes tratados en un ámbito multidisciplinar demuestran un mejor conocimiento de la ERC y mejores parámetros bioquímicos ${ }^{54}$.

Todos los estudios que comparan este tipo de atención con la estándar, proporcionada solamente por el nefrólogo, indican que fue más ventajosa en varios aspectos ${ }^{42}$, como el aumento en la supervivencia ${ }^{44}$.

A pesar de todas sus ventajas, pocos son los países que cuentan con un equipo multidisciplinario, compuesto por médicos, enfermeros, dietistas, psicólogos, entre otros. Según Mendelssohn ${ }^{55}$, hay dos barreras principales que evitan el uso de las consultas multidisciplinarias. La primera es la no aceptación por parte de los nefrólogos de que sea más ventajoso que la atención ofrecida por ellos. La segunda es la falta de infraestructura, espacio, personal y sueldo, destinando poco presupuesto a este fin, a pesar de que se ha demostrado que es más rentable porque disminuye los ingresos hospitalarios y retrasa la progresión de la enfermedad $38,48,56$.

Hay una gran cantidad de estudios que evalúan la educación y los resultados en pacientes que ya han comenzado tratamiento renal sustitutivo en diálisis, pero son necesarios más, que se centren en etapas más precoces de la enfermedad renal, como en la etapa prediálisis. A su vez la mayoría de estudios existentes sobre educación en la etapa prediálisis evalúan cómo afecta a la elección de tratamiento, mientras que se necesitarían más estudios que abarquen otras variables.

En general, los hallazgos de este estudio muestran resultados positivos para las intervenciones educativas en la etapa prediálisis, y se evidencia que hay un gran campo de actuación en este sentido, que está poco desarrollado y estudiado.

Durante la realización de este trabajo, la principal limitación que se ha presentado es la falta de artículos con resultados y evidencia sobre el tema tratado. 
A la vista de estos resultados, podemos concluir que, la implantación de una consulta prediálisis multidisciplinar, es hoy en día una necesidad de primer orden dentro de todo servicio de Nefrología, sirviendo de apoyo al médico en la realización de su trabajo y permitiendo una mayor adaptación del paciente al tratamiento y una optimización de los recursos.

La educación sanitaria se ha mostrado eficaz para aumentar el nivel de conocimientos que el paciente tiene sobre su enfermedad, tomar una decisión informada sobre la elección del tratamiento idóneo para él, iniciar la diálisis de forma programada, mejorar la tasa de mortalidad y morbilidad, disminuir los ingresos hospitalarios y mejorar el estado del paciente.

Dentro de la consulta prediálisis, es imprescindible la labor de enfermería a la hora de desarrollar los programas de educación sanitaria, para lo que debe estar formada como enfermera nefrológica y tener una línea de teléfono directa para poder contactar con los pacientes, y viceversa. Aunque en la actualidad esto no se realice, los profesionales deberían hacer un esfuerzo en conjunto para que haya una consulta prediálisis 0 ERCA en cada hospital.

\section{Agradecimientos}

A Rodolfo Crespo Montero, profesor titular de la Facultad de Medicina y Enfermería de la Universidad de Córdoba, como tutor de este trabajo.

Recibido: 30 Mayo 2014

Revisado: 2 Junio 2014

Modificado: 2 Junio 2014

Aceptado: 2 Junio 2014

\section{Bibliografía}

1. Alcázar R, Orte L, Otero A. Enfermedad renal crónica avanzada. Nefrología. 2008; 28 Supl 3: 3-6.

2. Otero LM. Enfermedad renal crónica. Colomb Med. 2002; 33 (1): 38-40.

3. Zamora I, Sanahuja MJ. Enfermedad renal crónica. AEP. 2008; 21: 231-239.

4. Coresh J, Selvin E, Stevens LA, Manzi J, Kusek JW, Eggers P, et al. Prevalence of chronic kidney disease in the United States. JAMA. 2007; 298 (17): 2038-2047.
5. Torres C. Insuficiencia renal crónica. Rev Med Hered. 2003; 14 (1): 1-4.

6. Otero A. Guías SEN para el manejo de la Enfermedad Renal Crónica Avanzada y Pre-Diálisis. Nefrología. 2008; Supl 3: 1.

7. Alcázar R, Egocheaga MI, Orte L, Lobos JM, González E, Álvarez F, et al. Documento de consenso SEN-semFYC sobre la enfermedad renal crónica. Nefrología. 2008; 28 Supl 3: 273-282.

8. Soriano S. Definición y clasificación de los estadios de la enfermedad renal crónica. Prevalencia. Claves para el diagnóstico precoz. Factores de riesgo de enfermedad renal crónica. Nefrología. 2004; 24 Supl 6: 27-34.

9. De Francisco $A L$, Otero A. Epidemiología de la enfermedad renal crónica en España. Nefrología. 2003; 23 (6): 475-477.

10. Górriz JL, Otero A. Impacto socio sanitario de la enfermedad renal crónica avanzada. Nefrología. 2008; 28 Supl 3: 7-15.

11. Bardón E, Marti A, Vila ML. Enfermería en la consulta de enfermedad renal crónica avanzada (ERCA). Nefrología. 2008; 28 Supl 3: 53-56.

12. Arias M, Alonso R, Menezo R, Escallada R: Educación del paciente en insuficiencia renal progresiva. En: Aljama P, Arias M, Valderrábano F, editores: Insuficiencia Renal Progresiva. Madrid: Grupo Entheos; 2000. p. 285-297.

13. Álvarez $\mathrm{R}$, Velasco $\mathrm{S}$. La consulta de prediálisis de enfermería: logros y oportunidades de mejora. Rev Soc Esp Enfermería Nefrológica. 2007; 10 (3): 106-171.

14. Tejedor A, Sanz F, Pérez de Lucas N, López R, García Y, López JM, et al. Atención domiciliaria al paciente urémico terminal no susceptible de diálisis. Nefrología. 2006; 26 Supl 3: 66-81.

15. Orte L, Barril G. Unidad de enfermedad renal crónica avanzada (ERCA). Concepto de una unidad multidisciplinaria. Objetivos de la consulta de ERCA. Nefrología. 2008; 28 Supl 3: 49-52.

16. Perea R. Educación para la salud, reto de nuestro tiempo. Educación XXI. 2001; 4: 15-40.

17. Rogero Anoya P. Educación para la salud en grupo. En: Frías A. Salud pública y educación para la salud. $1^{\circ}$ ed. Barcelona: Masson; 2000. p. 375-391. 
18. Barceló A, Robles S, White F, Jadue L, Vega J. Una intervención para mejorar el control de la diabetes en Chile. Rev Panam Salud Pública. 2001; 10 (5): 328-333.

19. Norris SL, Engelgau MM, Venkat KM. Effectiveness of Self-Management Training in Type 2 Diabetes. Diabetes Care. 2001; 24 (3): 561-587.

20. Pérez A, Barrios Y, Monier A, Berenguer M, Martínez I. Repercusión social de la educación diabetológica en personas con diabetes mellitus. Medisan [Internet]. 2009 [citado el 3 de Mayo de 2014]; 13 (1): 1-8. Disponible en: http://bvs.sld.cu/revistas/san/vol13_1_09/sanll109.htm

21. Gaede $\mathrm{P}$, Vedel P, Parving HH, Pedersen 0. Intensified multifactorial intervention in patients with type 2 diabetes mellitus and microalbuminuria: the Steno type 2 randomised study. The Lancet. 1999; 353: 617-622.

22. Norris $\mathrm{SL}$, Nichols $\mathrm{PJ}$, Caspersen $\mathrm{CJ}$, Glasgow RE, Engelgau M M, Jack $L$, et al. The effectiveness of disease and case management for people with diabetes. Am J Prev Med. 2002; 22 (4S): 15-38.

23. Aráuz AG, Sánchez G, Padilla G, Fernández M, Roselló M, Guzmán S. Intervención educativa comunitaria sobre la diabetes en el ámbito de la atención primaria. Rev Panam Salud Pública. 2001; 9 (3): 145-153.

24. González A, Martínez R. Efectividad de una estrategia educativa sobre los parámetros bioquímicos y el nivel de conocimientos en pacientes diabéticos tipo 2. Revista de Endocrinología y Nutrición. 2007; 15 (3): 165-174.

25. Ariza C, Gavara V, Muñoz A, Aguera $F$, Soto $M$, Lorca JR. Mejora en el control de los diabéticos tipo 2 tras una intervención conjunta: educación diabetológica y ejercicio físico. Aten Primaria. 2011; 43 (8): 398-406.

26. Borja C, Quintero JM, Sánchez R, Martínez C, Listerri R, Aizpiri R, et al. Resultado de los cambios de actitudes conseguidos a través de la EpS en diabéticos tipo II. Enfermería Integral. 2001; 56: XXI-XXVI.

27. Pérez MJ, Echauri M, Ancizu E, Chocarro J. Manual de educación para la salud. Navarra: Instituto de salud pública; 2006.

28. Renau $E$, Cerrillo V, Ribalta $C$, Folch MJ, Trilles AA, Mallol A, et al. Consulta de enfermedad renal crónica avanzada (ERCA) para toda una provincia. Enferm Nefrol. 2008; 33: 1-3.

29. Choi ES, Lee J. Effects of a face-to-face self-management program on knowledge, self-care practice and kidney function in patients with chronic kidney disease before the renal replacement therapy. J Korean Acad Nurs; 2012; 42 (7): 1070-1078.

30. Devins GM, Mendelssohn DC, Barré PE, Taub K, Binik YM. Predialysis psychoeducational intervention extends survival in CKD: A 20-Year FollowUp. Am J Kidney Dis. 2005; 46 (6): 1088-1098.

31. Portela L, Menéndez A, Bermúdez C, Mojón M, Morrondo J, Pérez MT. Influencia del nivel de conocimiento de los pacientes sobre la elección de las distintas opciones diálisis. Efecto de un protocolo de información reglada. Enferm Nefrol. 2001: 121-127.

32. Manns BJ, Taub J, VanderStraeten $C$, Jones $H$, Mills $C$, Visser M, et al. The impact of education on chronic kidney disease patients' plans to initiate dialysis with self-care dialysis: A randomized trial. Kidney International. 2005; 68: 1777-1783.

33. Yang LM, Han $T C$, Lin $P L$, Sun $C A$, Chou $Y C$, Chang $\mathrm{C}$, et al. Health knowledge and effectiveness of behavior improvement due to the intervention of healthcare programs for early stages of chronic kidney diseases. World Journal of Medicine and Medical Science [Internet]. 2013 [citado el 29 de Abril de 2014]; 1 (7): 136-152. Disponible en: http://www.wjmms.com/WJM MS_Vol.\%20 $1, \% 20$ No. $\% 207, \% 20$ November $\% 202013 /$ Health\%20Knowledge.pdf.

34. Marrón B, Ortiz A, de Sequera P, Martín-Reyes G, de Arriba G, Lamas JM, et al. Impact of endstage renal disease care in planned dialysis start and type of renal replacement therapy - a Spanish multicenter experience. Nephrol Dial Transplant. 2006; 21 Supl 2: 51-55.

35. Pulido F, Arribas E, Pulido JF, González F, Aragonzillo I. Tres años en la consulta ERCA. Enferm Nefrol. 2009; 34: 250-251.

36. Gutiérrez JM, Ibars P, Pitarch G. Evaluación de los conocimientos adquiridos en la consulta de prediálisis. Enferm Nefrol. 2002; 27: 180-186.

37. Devins GM, Mendelssohn DC, Barré PE, Binik YM. Predialysis psychoeducational intervention and coping styles influence time to dialysis in chronic kidney disease. Am J Kidney Dis. 2003; 42 (4): 693-703. 
38. Marrón B, Martínez JC, Salgueira M, Barril G, Lamas JM, Martín M, et al. Analysis of patient flow into dialysis: role of education in choice of dialysis modality. Perit Dial Int. 2005; 25 Supl 3: S56-S59.

39. Gago MC, Martínez S, Sesmero C, Andrés MM, Velayo $P$, Hernández $E$, et al. Influencia de la consulta predialisis en los pacientes con enfermedad renal crónica avanzada. Enferm Nefrol. 2007; 1 (32): 76-80.

40. Lacson E, Wang W, De Vries C, Leste K, Hakim RM, Lazarus M, et al. Effects of a Nationwide Predialysis Educational Program on Modality Choice, Vascular Access, and Patient Outcomes. Am J Kidney Dis. 2011; 58 (2): 235-242.

41. Wu IW, Wang SY, Hsu KH, Lee CC, Sun CY, Tsai CJ, et al. Multidisciplinary predialysis education decreases the incidence of dialysis and reduces mortality - a controlled cohort study based on NFK/ DOQI guidelines. Nephrol Dial Transplant. 2009; 24: 3426-3433.

42. Curtis BM, Ravani $P$, Maberti $F$, Kennett F, Taylor PA, Djurdjev 0, et al. The short and long term impact of multidisciplinary clinics in addition to standard nephrology care on patient outcomes. Nephrol Dial Transplant. 2005; 20: 147-154.

43. Gómez AC, Fernández D, 0jeda MA, Cabello C, Carcamo J, Ramírez MA. Impacto de la educación sanitaria en la elección de la modalidad de diálisis. Enferm Nefrol. 2011; 36: 24-29.

44. Goldstein M, Yassa T, Dacouris N, McFarlane P. Multidisciplinary Predialysis Care and Morbility and Mortality of Patients on Dialysis. Am J Kidney Dis. 2004; 44 (4): 706-714.

45. Goovaerts $T$, Jadoul M, Goffin E. Influence of a Pre-Dialysis Education Programme (PDEP) on the mode of renal replacement therapy. Nephrol Dial Transplant. 2005; 20: 1842-1847.

46. Levin A, Lewis M, Mortiboy P, Faber S, Hare I, Porter EC, et al. Multidisciplinary predialysis programs: quantification and limitations of their impact on patient outcomes in two Canadian settings. Am J Kidney Dis. 1997; 29 (4): 533-540.

47. Hemmelgarn BR, Manns BJ, Zhang J, Tonelli M, Klrenbach S, Walsh M, et al. Association between multidisciplinary care and survival for elderly patients with chronic kidney disease. J Am Soc Nephrol. 2007; 18: 993-998.
48. Jungers $P$, Massy ZA, Nguyen-Khoa $T$, Choukroun $G$, Robino C, Fakhouri F, et al. Longer duration of predialysis nephrological care is associated with improved long-term survival of dialysis patients. Nephrol Dial Transplant. 2001; 16: 2357-2364.

49. Harris LE, Luft FC, Rudy DW, Kesterson JG, Tierney WM. Effects of multidisciplinary case management in patients with chronic renal insufficiency. Am J Med. 1998; 105: 464-471.

50. White CA, Pilkey RM, Lam M, Holland DC. Predialysis clinic attendance improves quality of life among hemodialysis patients. BMC Nephrology. 2002; 3 (3): 1-8.

51. Rognant N, Alamartine E, Aldigier JC, Combe $C$, Vendrely $B$, Deteix $P$, et al. Impact of prior CKD management in a renal care network on early outcomes in incident dialysis patients: a prospective observational study. BMC Nephrology [Internet]. 2013 [citado el 30 de Abril de 2014]; 14: 41-51. Disponible en: http://www.biomedcentral. com/1471-2369/14/41.

52. Chen SH, Tsai YF, Sun CY, Wu IW, Lee CC, Wu MS. The impact of self-management support on the progression of chronic kidney disease - a prospective randomized controlled trial. Nephrol Dial Transplant. 2011; 26: 3560-3566.

53. Martín de Francisco AL, Aguilera L, Fuster V. Enfermedad cardiovascular, enfermedad renal y otras enfermedades crónicas. Es necesaria una intervención más temprana en la enfermedad renal crónica. Nefrología. 2009; 29 (1): 6-9.

54. Aguilera AI, Prieto M, González L, Abad B, Martínez E, Robles I, et al. Una estrategia poco utilizada en el cuidado de pacientes con enfermedad renal crónica: la educación en grupo y multidisciplinar de pacientes y sus familiares. Enferm Nefrol. 2012; 15 (1): 14-21.

55. Mendelssohn DC. Coping with the CKD epidemic: the promise of multidisciplinary team-based care. Nephrol Dial Transplant. 2005; 20: 10-12.

56. Arrieta J. Evaluación económica del tratamiento sustitutivo renal (hemodiálisis, diálisis peritoneal y trasplante) en España. Nefrología. 2010; 1 Supl 1: 37-47. 